\title{
Follow-up after Direct-acting Antiviral Treatment for Chronic Hepatitis C Virus Infection: Most Patients Are Followed Appropriately
}

\author{
Hiroki Tojima ${ }^{1}$, Satoru Kakizaki ${ }^{1,2}$, Satoshi Takakusagi ${ }^{3}$, Takashi Hoshino ${ }^{4}$, \\ Atsushi Naganuma ${ }^{4}$, Tamon Nagashima ${ }^{5}$, Masashi Namikawa ${ }^{6}$, Takashi Ueno ${ }^{7}$, \\ Yasushi Shimada $^{7}$, Takeshi Hatanaka ${ }^{8}$, Daichi Takizawa ${ }^{9}$, Hirotaka Arai ${ }^{9}$, Ken Sato ${ }^{1}$, \\ Hitoshi Takagi ${ }^{3}$ and Toshio Uraoka ${ }^{1}$
}

\begin{abstract}
:
Objective Chronic hepatitis $\mathrm{C}$ virus $(\mathrm{HCV})$ infection carries a residual risk of hepatocarcinogenesis even after viral elimination, so appropriate follow-up is necessary. The present study investigated the current hospital visits and hepatocarcinogenesis status of patients who received daclatasvir plus asunaprevir treatment (DCV+ ASV) to determine whether or not appropriate follow-up was being performed.

Methods We retrospectively analyzed hepatocarcinogenesis, the overall survival, and the length of hospital visits in 442 patients who applied for the medical expense subsidy system for viral hepatitis and received DCV+ASV treatment in Gunma Prefecture between October 2014 and December 2015. This also included 61 patients who had a history of hepatocellular carcinoma (HCC).

Results Among 442 patients, 388 achieved a sustained viral response (SVR) by DCV+ASV therapy (87.8\%), and 95.9\% achieved an SVR if additional treatment was included. HCC was found in 75 cases (17.0\%). A history of HCC, the FIB-4 index and the treatment effect SVR were determined to be factors affecting the incidence of HCC. Regarding the follow-up rate, $89.9 \%$ of patients continued to regularly visit the hospital after 5 years of treatment. However, patients $\leq 60$ years old had significantly lower persistence rates than older patients. The persistence rate of hospital visits to the same institution was $67.7 \%$ over a 5 -year period, which was significantly better in small and medium-sized institutions than in large, specialized institutions $(71.7 \%$ vs. $63.9 \%, \mathrm{p}=0.039)$.

Conclusion Patients with direct-acting antiviral treatment generally received adequate follow-up, but younger patients had a slightly higher rate of follow-up interruption and were considered to need support.
\end{abstract}

Key words: hepatitis C, retreatment, direct-acting antivirals, daclatasvir, asunaprevir, follow-up, hepatocarcinogenesis

(Intern Med 60: 3061-3070, 2021)

(DOI: 10.2169/internalmedicine.6591-20)

\footnotetext{
${ }^{1}$ Department of Gastroenterology and Hepatology, Gunma University Graduate School of Medicine, Japan, ${ }^{2}$ Department of Clinical Research, National Hospital Organization Takasaki General Medical Center, Japan, ${ }^{3}$ Department of Gastroenterology, Kusunoki Hospital, Japan, ${ }^{4}$ Department of Gastroenterology, National Hospital Organization Takasaki General Medical Center, Japan, ${ }^{5}$ Department of Gastroenterology, National Hospital Organization Shibukawa Medical Center, Japan, ${ }^{6}$ Department of Internal Medicine, Kiryu Kosei General Hospital, Japan, ${ }^{7}$ Department of Internal Medicine, Isesaki Municipal Hospital, Japan, ${ }^{8}$ Department of Gastroenterology, Gunma Saiseikai Maebashi Hospital, Japan and ${ }^{9}$ Department of Gastroenterology, Maebashi Red Cross Hospital, Japan
}

Received for publication October 26, 2020; Accepted for publication February 16, 2021

Correspondence to Dr. Hiroki Tojima, m08702015@gunma-u.ac.jp 


\section{Introduction}

Hepatitis $\mathrm{C}$ virus (HCV) infection causes liver fibrosis and is associated with a high risk of hepatocarcinogenesis (1). Although interferon (IFN) therapy was widely used for hepatitis $\mathrm{C}$ treatment in the past, oral IFN-free treatment of hepatitis $\mathrm{C}$ with direct-acting antiviral agents (DAAs) has been possible since 2014, and a sustained viral response (SVR) can now be achieved with even higher rates.

Grecaprevir/pibrentasvir combination therapy, a mainstay treatment regimen for $\mathrm{HCV}$ infection at present, has been reported to result in a cure in approximately $99 \%$ of chronic hepatitis $\mathrm{C}$ patients in a real world clinical setting and to be highly effective even in cases of unsuccessful DAA treatment (2-7). In IFN treatment, an SVR reduces the risk of hepatocellular carcinoma (HCC), but the risk of hepatocarcinogenesis itself is known to remain (8-13). Although the risk of hepatocarcinogenesis after viral exclusion by DAAs has been controversial $(14,15)$, it was recently reported that the SVR induced by DAAs as well as by IFN reduced the hepatocarcinogenesis rate (16-19). Furthermore, an SVR induced by DAAs reduced not only the risk of hepatocarcinogenesis but also that of progression to decompensated cirrhosis, liver disease-related death, and all-cause mortality (20). Given the above, DAA treatment may improve the prognosis of patients with chronic hepatitis $\mathrm{C}$.

However, there have been cases of hepatocarcinogenesis long after SVR in some patients previously treated with IFN therapy (12), and a similar phenomenon can be expected in cases treated with DAA. Therefore, appropriate follow-up and regular liver cancer screening is necessary after treatment is completed. Combination therapy with daclatasvir plus asunaprevir (DCV+ASV) was the first IFN-free, all-oral DAA treatment available in Japan, introduced in 2014, and a clinical trial showed this regimen to have high therapeutic efficacy, with an SVR rate after 24 weeks (SVR24) of $84.7 \%(21,22)$. This treatment allowed more patients to achieve an SVR than before, including those who had previously been intolerant of IFN.

We also reported that patients who failed to achieve an SVR with DCV+ASV were able to achieve an SVR at a high rate with additional DAA treatments (23). DCV+ASV has been available for over five years now, which has allowed for the long-term observation of treated cases; however, reports with appropriate follow-up are still lacking.

The present study investigated the current status of DCV+ ASV-treated patients (persistence of hospital visits and progression of cancer) in a retrospective manner to determine whether or not patients treated with DAA are being followed up appropriately and to investigate any relevant issues.

\section{Materials and Methods}

\section{Patients and treatment methods}

Between October 2014 and December 2015, there were 641 applications for DCV+ASV treatment using the medical expense subsidy system for viral hepatitis in Gunma Prefecture. Of these, we reviewed 442 cases treated at Gunma University Hospital and related institutions with DCV+ASV treatment. DCV+ASV treatment was usually continued for 24 weeks. Treatment institutions were divided into two groups: small and medium-sized institutions with $<300$ inpatient beds (Group A), which included non-bed clinics, and large, specialized institutions with $\geq 300$ inpatient beds (Group B). These data include cases of both liver cirrhosis and chronic hepatitis as well as those with a history of HCC. The data were obtained from a survey of medical records at each institution.

This study was conducted in accordance with the ethical guidelines of the 1975 Declaration of Helsinki and was approved by the Ethics Committee of the Gunma University Hospital. It was a retrospective study, and the need for written informed consent was waived.

\section{Patients" data}

Laboratory data included the aspartate aminotransferase (AST, IU/L), alanine aminotransferase (ALT, IU/L) levels; the platelet count $\left(\times 10^{4} / \mu \mathrm{L}\right)$; and the HCV-RNA value at the start of treatment. The HCV-RNA at 24 weeks after the end of treatment was used to determine the SVR. The HCVRNA (log copies/mL) was measured by real-time reverse transcription polymerase chain reaction (RT-PCR), as were the HCV genotypes (BML, Tokyo, Japan). The fibrosis-4 (FIB-4) index was calculated as follows: [AST (IU/L) $\times$ age (years)]/[platelet count $\left.\left(\times 10^{4} / \mu \mathrm{L}\right) \times \sqrt{ }(\mathrm{ALT}(\mathrm{IU} / \mathrm{L}))\right](24)$.

\section{Treatment method}

Each patient was administered $60 \mathrm{mg}$ of DCV and 100 $\mathrm{mg}$ of ASV orally once a day in accordance with the Japanese Society of Hepatology guidelines at the time. If the patients showed good tolerance, they were usually treated for 24 weeks. The efficacy of treatment was determined by the SVR24.

\section{Follow-up}

The survey was conducted as of February 2020. We defined the duration of continuous hospital visits to the same institution as the time between the start of treatment and referral to another hospital. The duration of continuous hospital visits was defined as the time between the start of treatment and the interruption of hospital visits (no follow-up). The date of the onset of HCC after the start of treatment was investigated in all patients. The onset of HCC was determined by the characteristic findings on imaging [computed tomography (CT) and magnetic resonance imaging 
Table 1. Characteristics of Patients with Hepatitis C Virus who were Treated with DCV+ASV $(n=442)$.

\begin{tabular}{lcccc}
\hline \multicolumn{1}{c}{ Characteristics } & & Group A institutions & Group B institutions & p value \\
\hline All patients & 442 & 218 & 224 & \\
Age & $68[62,73]$ & $68[61,74]$ & $68[63,73]$ & 0.924 \\
Sex & & & & 0.848 \\
$\quad$ Male & $200(45.2)$ & $100(45.9)$ & $100(44.6)$ & \\
$\quad$ Female & $242(54.8)$ & $118(54.1)$ & $124(55.4)$ & \\
Treatment history for HCC & & & $178(79.5)$ & \\
$\quad$ Absent & $381(86.2)$ & $203(93.1)$ & $46(20.5)$ & \\
$\quad$ Present & $61(13.8)$ & $15(6.9)$ & & 0.001 \\
Compesated Cirrhosis & & & $131(58.7)$ & \\
$\quad$ Absent & $289(65.5)$ & $158(72.5)$ & $92(41.3)$ & \\
$\quad$ Present & $152(34.5)$ & $60(27.5)$ & $6.2[5.7,6.4]$ & 0.532 \\
HCVRNA (log copy/mL) & $6.1[5.6,6.5]$ & $6.1[5.6,6.5]$ & & 0.531 \\
HCV serogroup or genotype & & & $156(69.6)$ & \\
$\quad$ serogroup 1 & $314(71.0)$ & $158(72.5)$ & $68(30.4)$ & \\
genotype $1 b$ & $128(29.0)$ & $60(27.5)$ & $51.0[38.0,70.0]$ & 0.048 \\
AST (U/L) & $49.0[35.0,68.0]$ & $45.0[33.0,63.0]$ & $44.0[30.0,66.5]$ & 0.506 \\
ALT (U/L) & $43.0[29.0,63.3]$ & $43.0[29.0,63.0]$ & $11.5[8.5,15.0]$ & $<0.001$ \\
Platelet count $\left(\times 10^{4} / \mathrm{uL}\right)$ & $12.5[8.9,17.1]$ & $13.8[9.7,18.2]$ & $4.50[3.12,6.61]$ & $<0.001$ \\
FIB-4 index & $4.08[2.64,6.35]$ & $3.55[2.28,5.85]$ & &
\end{tabular}

Data are expressed as the median [interquartile range] or count (percentage)

HCC: hepatocellular carcinoma, AST: aspartate aminotransferase, ALT: alanine aminotransferase, FIB-4: fibrosis-4

(MRI)] (25). The date of death and presence of liver disease-related death were also investigated in all patients who died.

\section{Statistical analyses}

The continuous variables are presented as the median [interquartile range] and nominal variables as the number (percentage). Comparisons of continuous variables between groups were made with the Mann-Whitney U test. Comparisons of nominal variables were made with the chi-square test or Fisher's exact test. The overall survival, duration of continuous hospital visits to the same institution, duration of continuous hospital visits, and time to hepatocarcinogenesis were analyzed using the Kaplan-Meier method, and generalized Wilcoxon's or Log-rank tests were used for group comparisons. A Cox proportional hazards analysis was used to adjust for covariates when analyzing the interruption of hospital visits to the same institution and time to carcinogenesis. The independent variables were selected based on the results of the univariate analysis and those considered necessary according to previous reports.

The IBM Statistical Package for the Social Sciences software program, version 25 (IBM SPSS 25; IBM, Armonk, USA) and EZR (26) were used for these statistical analyses. All $\mathrm{p}$ values were considered statistically significant at $<$ 0.05 .

\section{Results}

\section{Patients' background characteristics}

Patients' background characteristics are shown in Table 1. Two hundred and eighteen of 442 patients (49.3\%) were treated at group A institutions. The median age was 68 [interquartile range, 62.3-73.3] years old, and 200 patients were men. There were 61 patients with a history of HCC treatment. One hundred and fifty-two patients were clinically diagnosed with compensated cirrhosis, but no cases of decompensated cirrhosis were included. Patients treated at Group A institutions were significantly less likely to have a history of HCC (Group A: 6.9\%, Group B: 20.5\%, p<0.001) and significantly less likely to have compensated liver cirrhosis (Group A: 27.5\%, Group B: 41.3\%, p=0.003) than Group B patients. Blood test results showed that Group A had significantly lower AST than Group B institutions (Group A: 45.0 IU/l, Group B: 51.0 IU/1, p=0.048) and preserved platelet counts (Group A: $13.8 \times 10^{4} / \mu \mathrm{L}$, Group B: $\left.11.5 \times 10^{4} / \mu \mathrm{L}, \mathrm{p}<0.001\right)$. Similarly, the FIB-4 index was better in Group A than in Group B (Group A: 3.55, Group B: 4.50, $\mathrm{p}<0.001)$.

\section{Outcomes}

The treatment effect, hospital visits, survival, and hepatocarcinogenesis status are described in Table 2. An SVR24 was achieved in $87.8 \%$ of cases (388 of 442) with DCV+ ASV treatment, which was comparable to clinical trials (21). There was no marked difference in the treatment effect of 
Table 2. Outcome of DCV+ASV Treated Patients $(n=442)$.

\begin{tabular}{|c|c|c|c|c|}
\hline Results & & Group A institutions & Group B institutions & $\mathrm{p}$ value \\
\hline All patients & 442 & 218 & 224 & \\
\hline Status of hospital visits & & & & 0.030 \\
\hline Continuing & $306(69.2)$ & $160(73.4)$ & $146(65.2)$ & \\
\hline Referred to another institutions & $96(21.7)$ & $36(16.5)$ & $60(26.8)$ & \\
\hline Interruption & $40(9.0)$ & $22(10.1)$ & $18(8.0)$ & \\
\hline Attributes of the referral institution & & & & 0.399 \\
\hline Specilized Hospital & $49(50.0)$ & $20(57.1)$ & $29(46.0)$ & \\
\hline General hospital \& Clinic & $49(50.0)$ & $15(42.9)$ & $34(54.0)$ & \\
\hline Death & $16(3.6)$ & $9(4.1)$ & $7(3.1)$ & 0.619 \\
\hline Liver disease-related death & $10(2.3)$ & $5(2.3)$ & $5(2.2)$ & 1.000 \\
\hline Treatment outcome & & & & 0.384 \\
\hline not SVR & $54(12.2)$ & $30(13.8)$ & $24(10.7)$ & \\
\hline SVR & $388(87.8)$ & $188(86.2)$ & $200(89.3)$ & \\
\hline Additional treatment of DAAs\$ & & & & 0.526 \\
\hline Absent & $14(26.0)$ & $8(26.7)$ & $6(25.0)$ & \\
\hline Present & $40(74.0)$ & $22(73.3)$ & $18(75.0)$ & \\
\hline Treatment outcome of additional treatment & & & & 0.114 \\
\hline not SVR & $4(10.0)$ & $4(18.2)$ & $0(0.0)$ & \\
\hline SVR & $36(90.0)$ & $18(81.8)$ & $18(100.0)$ & \\
\hline Development of new HCC\$ & & & & 0.005 \\
\hline Absent & $356(82.6)$ & $182(87.9)$ & $174(77.7)$ & \\
\hline Present & $75(17.4)$ & $25(12.1)$ & $50(22.3)$ & \\
\hline
\end{tabular}

DCV+ASV between Group A and B institutions (Group A: $86.2 \%$, Group B: $89.3 \%, \mathrm{p}=0.384$ ). Forty of 54 cases who did not achieve an SVR were given additional treatment with other DAAs, and 36 patients $(90.0 \%)$ achieved an SVR 24. Including additional treatments, 424 of 442 patients finally achieved an SVR (95.9\%), and there was no marked difference in the overall outcome between Group A and B institutions (Group A: 94.5\%, Group B: 97.3\%, p=0.154).

\section{New incidence of HCC}

Between the start of DCV+ASV and the time point of observation, the new incidence of HCC was observed in 75 patients. The cumulative incidence of HCC for all cases (Fig. 1a) was $4.2 \%$ at 1 year, $12.0 \%$ at 3 years, and $21.7 \%$ at 5 years. The cumulative incidence of HCC was $14.1 \%$ over 5 years in the group with no history of HCC prior to DCV+ASV treatment, compared to $62.9 \%$ in the group with such a history (Fig. 1b) $(\mathrm{p}<0.001)$. The cumulative incidence of HCC increased in patients with an FIB-4 index of $>3.25$. It was $8.2 \%$ over 5 years in the group with an FIB-4 index $\leq 3.25$ but $26.4 \%$ in the group with an FIB-4 index $>3.25$ (p $=0.009$, Fig. 1c). The cumulative hepatocarcinogenesis rate at 5 years in the group with a treatment effect of an SVR was $20.1 \%$ compared to $32.7 \%$ in the non-SVR group ( $\mathrm{p}=$ 0.036 , Fig. 1d). There was no significant difference in cumulative cancer rates between genders $(p=0.061$, Fig. 1e) and by age ( $\leq 60$ vs. $>60$ years old; $p=0.068$, Fig. $1 f)$.

A multivariate analysis was performed to adjust for co- variates. The independent variables entered into the COX proportional hazards model were as follows: treatment effect, FIB-4 index, history of HCC, age and gender. The analysis showed that a history of HCC [hazard ratio (HR): 7.183, 95\% confidence interval (CI): 4.40-11.72, $\mathrm{p}<0.001]$ and the FIB-4 index (HR: 1.060, 95\% CI: 1.008-1.116, p= $0.024)$ were factors contributing to hepatocarcinogenesis after DAA treatment (Table 3).

\section{Hospital visits}

The persistence of hospital visits was analyzed by the Kaplan-Meier method. The overall persistence rate for hospital visits was $100 \%$ at 1 year, $91.1 \%$ at 3 years, and $89.9 \%$ at 5 years, and most patients continued to visit hospitals (Fig. 2a). There was no marked difference in the duration of hospital visits in Groups A and B ( $p=0.609$, generalized Wilcoxon's test) (Fig. 2b). An age $>60$ years old was detected as a factor associated with longer hospital visits in the univariate analysis ( $\mathrm{p}=0.004)$ (Fig. 2c). A Cox proportional hazards test was performed with age $>60$ years old, an FIB-4 index $>3.25$, and a history of HCC as independent variables and showed that an age $>60$ years old was a significant factor for the interruption of hospital visits (HR= 0.372, 95\% CI 0.190-0.726, p=0.004) (Table 4). Hospital visits were interrupted during the observation period in 40 cases, including 25 cases of advanced fibrosis with an FIB-4 index $>3.25$ and 3 cases with a history of HCC (Table 5).

The overall persistence rate for hospital visits of same in- 

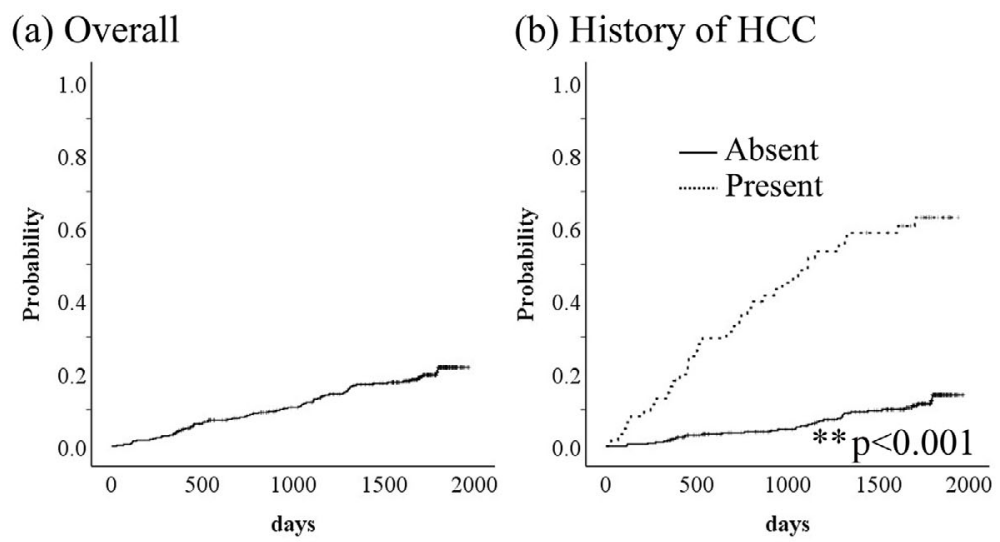

(c) FIB-4 index

(d) Treatment effect

(e) Gender
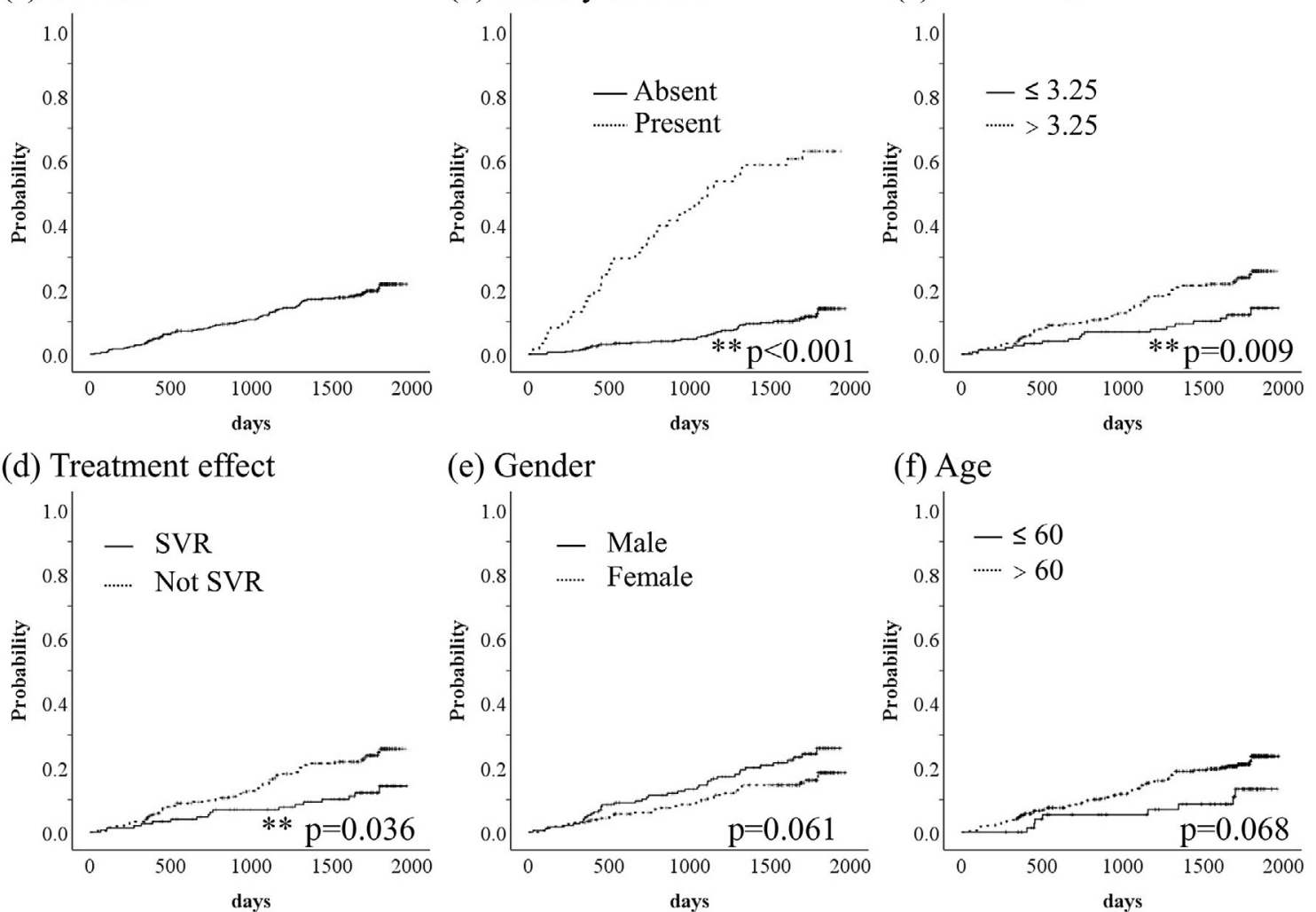

Figure 1. Cumulative incidence curve for hepatocellular carcinoma (HCC) after DCV+ASV treatment. (a) All of the patients. The 1-, 3-, and 5-year cumulative incidence rates of HCC were 4.2\%, $12.0 \%$, and $21.7 \%$, respectively. (b) Patients with or without a history of HCC. The 1-, 3-, and 5-year cumulative incidence rates of $\mathrm{HCC}$ were $1.9 \%, 5.6 \%$, and $14.1 \%$ in patients without a history of $\mathrm{HCC}$ and $16.4 \%, 48.4 \%$, and $62.9 \%$ in patients with a history of $\mathrm{HCC}$, respectively (p<0.001). (c) FIB-4 index $>3.25$ or $\leq 3.25$. The 1-, 3-, and 5-year cumulative incidence rates of $\mathrm{HCC}$ were $2.6 \%$, $6.9 \%$, and $14.3 \%$ in the FIB-4 index $\leq 3.25$ patients and $4.7 \%, 14.9 \%$, and $25.7 \%$ in the FIB-4 index $>3.25$ patients, respectively ( $p=0.009$ ). (d) Treatment effect of an SVR or not SVR. The 1-, 3-, and 5-year cumulative incidence rates of HCC were $5.6 \%, 18.2 \%$, and $32.7 \%$ in the non-SVR patients and $3.7 \%$, $11.1 \%$, and $20.1 \%$ in the SVR patients, respectively $(\mathrm{p}=0.036)$. (e) Men or women. The 1-, 3-, and 5 -year cumulative incidence rates were $4.7 \%, 15.6 \%$, and $25.9 \%$ in men, respectively, and $3.4 \%$, $10.0 \%$, and $18.3 \%$ in women, respectively $(p=0.061)$. (f) Age $\leq 60$ years old or $>60$ years old. The 1-, 3-, and 5-year cumulative incidence rates were $0 \%, 5.1 \%$, and $14.4 \%$ for those $<60$ years old, respectively, and $4.9 \%, 13.6 \%$, and $23.3 \%$ for those $>60$ years old, respectively (p=0.068). HCC: hepatocellular carcinoma, FIB-4: fibrosis-4, SVR: sustained viral response

Table 3. A Multivariate Analysis of Risk Factors for Newly Developed HCC.

\begin{tabular}{lccc}
\hline & Hazard ratio & $95 \%$ CI & p value \\
\hline Past history of HCC & 7.183 & $4.40-11.72$ & $<0.001$ \\
FIB-4 index & 1.060 & $1.008-1.116$ & 0.024 \\
Treatment effect SVR & 0.573 & $0.321-1.025$ & 0.061 \\
Age & 1.025 & $0.993-1.059$ & 0.126 \\
Gender : female & 0.930 & $0.574-1.508$ & 0.769 \\
\hline
\end{tabular}

HCC: hepatocellular carcinoma, FIB-4: fibrosis-4, SVR: sustained viral response, $\mathrm{CI}$ : confidence interval

stitution were $97.3 \%$ for 1 year, $82.5 \%$ for 3 years, and $67.7 \%$ for 5 years (Fig. 3a). The persistence rate for hospital visits of the same institution was $71.7 \%$ in Group A but
63.9\% in Group B at 5 years (Fig. 3b). Group A institutions had significantly higher persistence rates for visits to the same institution than Group B $(p=0.039$, generalized Wilcoxon's test). A univariate analysis detected a treatment history of $\mathrm{HCC}$ as another factor affecting the persistence rate for hospital visits to the same institution ( $\mathrm{p}=0.009)$ (Fig. 3c). A multivariate analysis was performed. The independent variables entered into the Cox proportional hazards model were as follows: a history of HCC, age $>60$ years old, an FIB-4 index $>3.25$, and institution grouping. The model showed that a history of HCC (HR $=0.406$, 95\% CI 0.217 0.758, $\mathrm{p}=0.005)$ and Group B institutions $(\mathrm{HR}=1.530,95 \%$ CI $1.085-2.185, \mathrm{p}=0.015$ ) were significant factors for the interruption of hospital visits to the same institution (Table 6). 

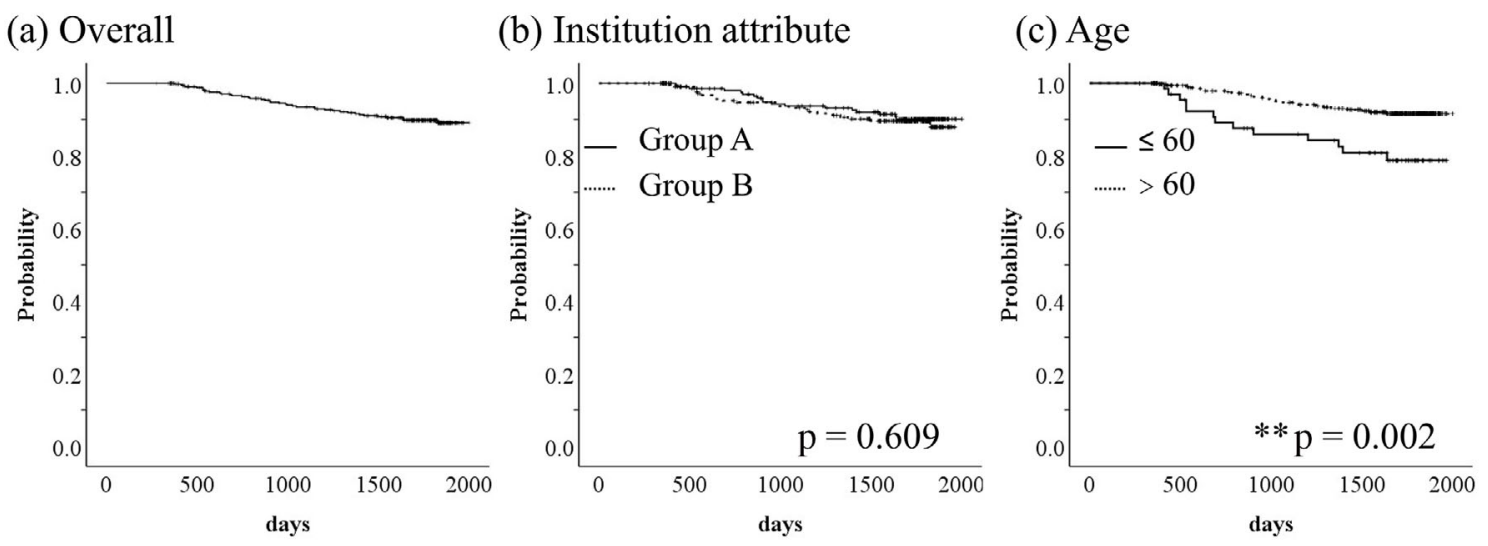

(d) FIB-4 index

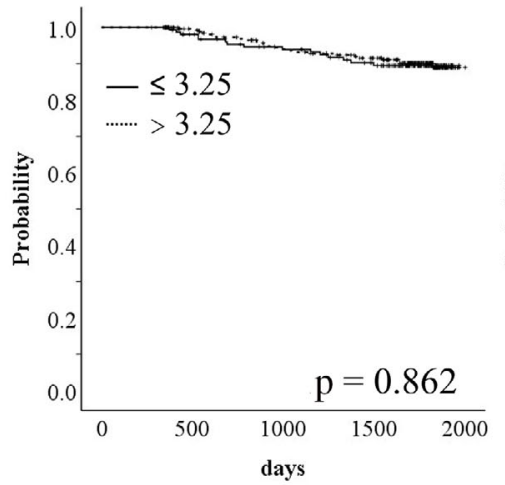

(e) History of HCC

(f) Treatment effect
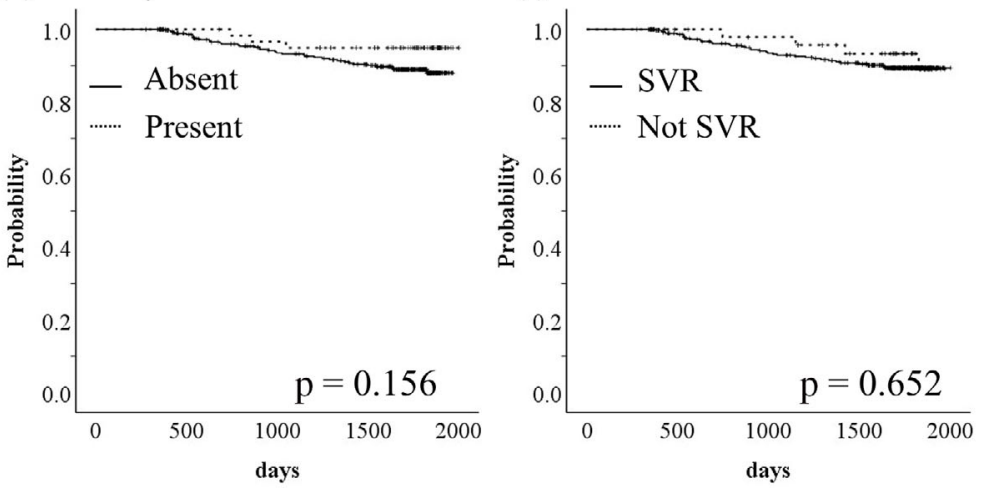

Figure 2. Persistence rate for hospital visits. (a) All patients. The 1-, 3-, and 5-year persistence rates for hospital visits were $100 \%, 93.5 \%$, and $89.9 \%$, respectively. (b) Institution attribute Group A or B. There was no significant difference between Group $A$ and $B$ (p=0.609). (c) Age $\leq 60$ or $\geq 60$ years old. The 1-, 3-, and 5-year persistence rates for hospital visits were $100 \%, 88 \%$, and $82 \%$ in patients $\leq 60$ years old, respectively, and $100 \%, 94.7 \%$, and $91.7 \%$, in patients $>60$ years old, respectively $(p=0.002)$. (d), (e), (f) There were no significant difference between those with an FIB-4 index $\leq 3.25$ and $>3.25$, no or some history of HCC, and a treatment effect of SVR and no SVR. FIB-4: fibrosis-4, HCC: hepatocellular carcinoma, SVR: sustained viral response

Table 4. A Multivariate Analysis of Interruption of Hospital Visits.

\begin{tabular}{lccc}
\hline & Hazard ratio & $95 \%$ CI & p value \\
\hline Age $>60$ & 0.372 & $0.190-0.726$ & 0.004 \\
FIB-4 index & 1.007 & $0.922-1.100$ & 0.873 \\
Past history of HCC present & 0.511 & $0.156-1.677$ & 0.268 \\
\hline
\end{tabular}

FIB-4: fibrosis-4, HCC: hepatocellular carcinoma, CI: confidence interval

\section{The survival}

There were 16 deaths at the time of observation, of which 10 were liver disease-related deaths. Factors affecting the survival included an FIB-4 index $>3.25$ ( $\mathrm{p}=0.048$ ) (Fig. 4a), a history of HCC ( $\mathrm{p}=0.013)$ (Fig. 4b), and a treatment effect of an SVR $(\mathrm{p}<0.001)$ (Fig. 4c) in the univariate analysis. Factors associated with liver disease-related death were a treatment effect of an SVR (p=0.007) (Fig. 5a) and a history of HCC ( $\mathrm{p}=0.002)$ (Fig. 5b). A multivariate analysis was not performed because there were few deaths, including liver disease-related deaths.

\section{Discussion}

The effect of treatment was generally favorable, with no marked difference between Group B (specialized institutions) and Group A (general institutions). In patients who failed DCV+ASV, there was no significant difference between Group A and Group B in the rate of additional DAA treatment and the final outcome. This suggests that DAAs are highly effective and their management appropriate, with no marked difference between institutions. Although DAA treatment is expensive, access to medical care for hepatitis $\mathrm{C}$ is good in Japan because of the development of medical ex- 
Table 5. Background of the Case of Interruption of Hospital Visit $(n=40)$.

\begin{tabular}{lc}
\hline \multicolumn{2}{c}{ Characteristics } \\
\hline All patients & 40 \\
Age & $67[37,83]$ \\
Sex & $22(55.0)$ \\
$\quad$ Male & $18(45.0)$ \\
$\quad$ Female & \\
Treatment history for HCC & $37(92.5)$ \\
$\quad$ Absent & $3(7.5)$ \\
$\quad$ Present & $28(70.0)$ \\
Compesated cirrhosis & $12(30.0)$ \\
$\quad$ Absent & $50.0[11.0,210.0]$ \\
$\quad$ Present & $52.0[13.0,155.0]$ \\
AST (U/L) & $12.5[8.9,17.1]$ \\
ALT (U/L) & $3.93[0.91,21.41]$ \\
Platelet count $\left(\times 10^{\wedge} 4 / \mathrm{uL}\right)$ & \\
FIB-4 index & $15(37.5)$ \\
FIB-4 index & $25(62.5)$ \\
$<3.25$ & \\
$>3.25$ & \\
\hline Data are expressed as the median [interquartile range] or \\
count (percentage) \\
AST: aspartate aminotransferase, ALT: alanine amino- \\
transferase
\end{tabular}

pense subsidy system for viral hepatitis and the coverage of DAA treatment since 2014 (27). In this study, 82 of 218 patients $(37.6 \%)$ in the Group A institutions were treated by general practitioners, and a wide base of patients was treated.

To monitor hepatocarcinogenesis from hepatitis $\mathrm{C}$, patients should continue to attend the hospital after $\mathrm{HCV}$ has been eliminated. It has been suggested that patients should be followed up for HCC by an imaging examination at least twice a year after SVR (28), and appropriate follow-up is especially important in groups at high risk for hepatocarcinogenesis. Indeed, 75 cases of HCC were found in the present study during the observation period, and about half of them (37/75) had a history of HCC. The incidence of HCC after DAA treatment was significantly higher in the patients with a history of HCC than in those without such a history. Advanced liver fibrosis (FIB-4 index >3.25) was also shown to be a significant factor influencing hepatocarcinogenesis after treatment. Previously reported factors contributing to hepatocarcinogenesis after SVR include advanced liver fibrosis, male gender, an advanced age, high pre-treatment alfa-fetoprotein (AFP) levels, an impaired glucose tolerance, dyslipidemia, an excessive alcohol intake, and high gamma-glutamyl transpeptidase levels $(11,12,16,29)$. Although the information available in this study was limited and not sufficient to assess the risk of carcinogenesis, the results are similar to those included in previous reports within the scope of the study, indicating the importance of appropriate follow-up in high-risk carcinogenesis groups.
In this study, the persistence rate of hospital visits was approximately $90 \%$ over a 5-year period (Group A: $89.6 \%$, Group B: $90.1 \%$, respectively), and although patients generally continued to be hospitalized in some form, the duration of hospital visits was significantly shorter among those $<60$ years old than in older patients. Darvishian et al. identified the following factors as more likely to cause loss of followup: age <60 years old, injection drug use (IDU), on opioid substitution therapy, and cirrhosis (30). They also cited high rates of IDU as one reason for the low rates of persistence of hospital visits among young people. Patients younger than 60 years old in the present study also tended to have significantly shorter hospital visits than older patients, but IDU was not assessed. However, the frequency of IDU in Japan is reported to be low compared to other countries (31). The HCV genotype of IDU patients in Japan is reportedly more common other than genotype 1 (32). However, since the present study included patients receiving combination therapy with DCV+ASV, all cases analyzed were genotype 1 . Therefore, it is unlikely that IDU was a major factor affecting the duration of hospital visits in the present study. In addition to the fact that young patients have high levels of social activity and find it difficult to visit regularly, the number of patients with relatively mild fibrosis and less severe complications may have influenced the duration of outpatient visits, and providing assistance, such as employment support, to patients with high social activity will likely continue to be an issue. For workers, it is considered important to introduce them to institutions where they can be examined on a schedule that fits with their working style and occupation. Regarding the financial burden associated with hospital visits, Japan has established a system to subsidize the cost of periodic examinations, and the cost of up to two examinations per year is subsidized. Publicizing this system is expected to reduce the financial burden associated with regular follow-up and lead to continued visits to the hospital. To support these measures, the employment support model was started in FY2014. In addition, guidance to support measures by hepatitis medical coordinators may be useful (33). In the Japanese medical system, most regional core hospitals are positioned as "special functioning hospitals" (https://www.mhlw.go.jp/english/wp/wp-hw3/dl/2027.pdf) or "regional medical care support hospitals" (http s://www.mhlw.go.jp/english/wp/wp-hw3/dl/2-028.pdf) and play a role as the core of regional medical care cooperation, providing advanced medical services and patients referred from regional medical institutions. These institutions are required to play a role in regional medical care by receiving referrals from regional medical institutions for patients who need advanced treatment and conversely refer patients who no longer need advanced treatment to regional medical institutions. In this study, we classified institutions with fewer than 300 beds and non-bed clinics as Group A institutions and those with 300 beds or more as Group B institutions. Most of the Group B institutions were special functioning hospitals or regional medical care support hospitals or 

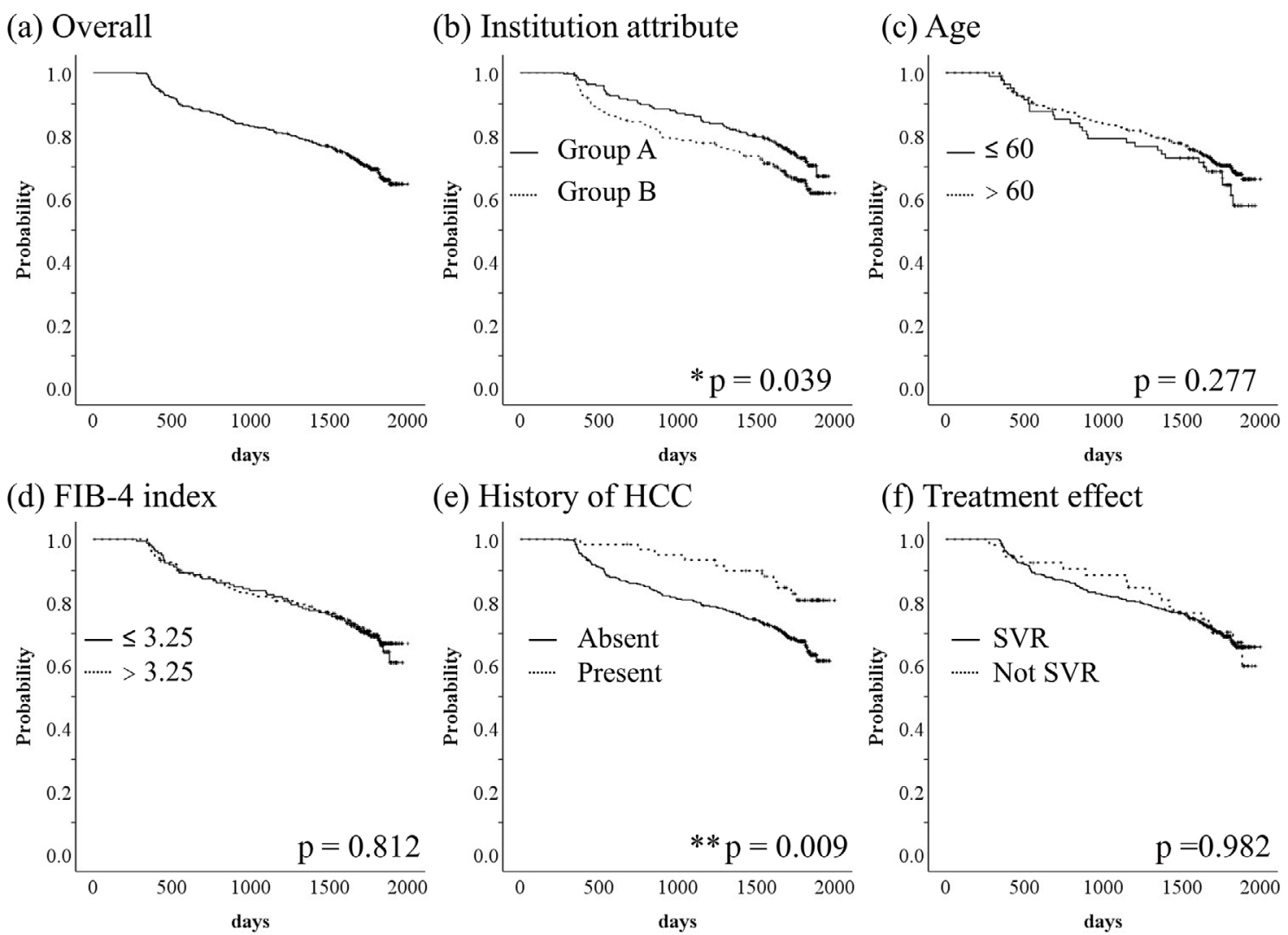

Figure 3. Persistence rate for hospital visits of same institution. (a) All patients. The 1-, 3-, and 5-year persistence rates for hospital visits to the same institution were $97.5 \%, 82.5 \%$, and $67.7 \%$, respectively. (b) Institution attribute. The 1-, 3-, and 5-year persistence rates for hospital visits to the same institution were 98.6\%, 86.6\%, and 71.7\% in Group $A$ and 96.0\%, 78.5\%, and 63.9\% in Group $B$, respectively $(\mathrm{p}=0.039)$. (c) Age $\leq 60$ years old or $>60$ years old. There was no significant difference between subjects $\leq 60$ years old or $>60$ years old. (d) FIB-4 index $\leq 3.25$ and $>3.25$. There was no significant difference between subjects with an FIB-4 index $\leq 3.25$ and $>3.25$. (e) Patients with or without a history of HCC. The 1-, 3-, and 5-year persistence rates for hospital visits to the same institution were $96.1 \%, 80.7 \%$, and $65.6 \%$ in patients without a history of $\mathrm{HCC}$ and $100 \%, 93.4 \%$, and $80.6 \%$ in patients with a history of $\mathrm{HCC}$, respectively ( $\mathrm{p}=0.009)$. (f) Treatment effect of SVR and no SVR. There was no significant difference between subjects with and without an SVR. FIB-4: fibrosis-4, HCC: hepatocellular carcinoma, SVR: sustained viral response

Table 6. A Multivariate Analysis of the Interruption for Hospital Visits to the Same Institution.

\begin{tabular}{lccc}
\hline & Hazard ratio & $95 \%$ CI & p value \\
\hline Past history of HCC & 0.409 & $0.218-0.765$ & 0.005 \\
Age $>60$ & 0.800 & $0.530-1.209$ & 0.289 \\
FIB-4 index & 1.002 & $0.955-1.052$ & 0.937 \\
Institution attribute Group B & 1.530 & $1.085-2.185$ & 0.015 \\
\hline
\end{tabular}

HCC: hepatocellular carcinoma, FIB-4: fibrosis-4, CI: confidence interval

equivalent institutions. Except for those patients at a very high risk of new hepatocarcinogenesis, many patients who completed the DAA treatment did not require advanced medical care for the management of their liver disease and were thus referred to other community hospitals after the response to treatment was confirmed. Indeed, the patients with a history of HCC had a significantly higher follow-up rate at the same institution and were more likely to be treated at a specialized institution than small or medium-sized institutions. This suggests that many of the patients with a history of HCC were adequately followed up at specialized centers. In the present study, DAA treatment was found to be widely performed in Group A institutions (general medical institutions), and appropriate follow-up was provided afterwards. In Japan, regional core centers for the treatment of liver disease were established in each of the 47 prefectures between 
(a) FIB-4 index

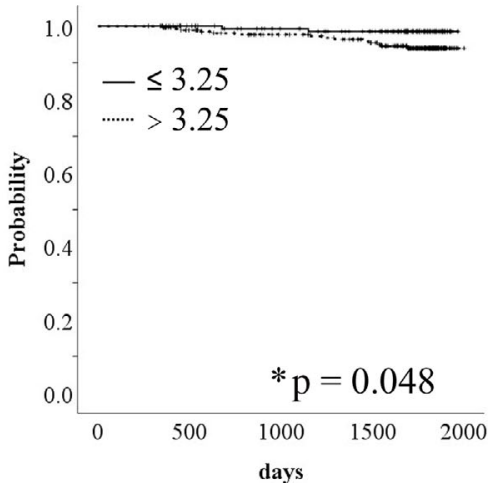

(b) History of HCC

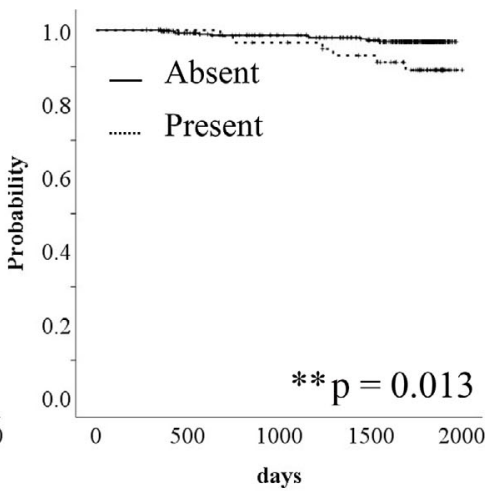

(c) Treatment effect

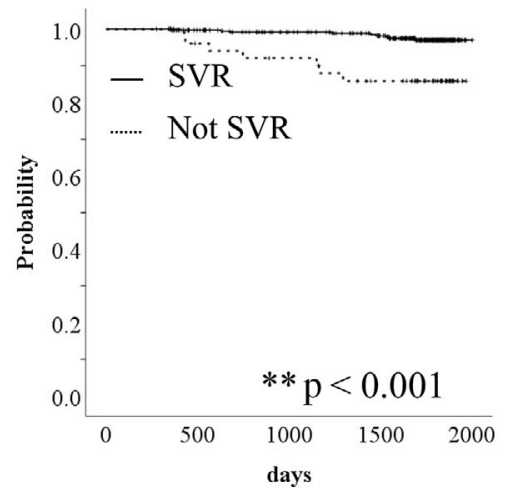

Figure 4. The overall survival. (a) The overall survival was significantly longer in the FIB-4 index $\leq 3.25$ group than in the $>3.25$ group $(p=0.048)$. (b) The overall survival was significantly longer in the patients without a history of HCC ( $p=0.013$ ). (c) The group that obtained an SVR with DCV+ASV therapy had a significantly longer overall survival than the group without an SVR (p<0.001). FIB-4: fibrosis-4, HCC: hepatocellular carcinoma, SVR: sustained viral response

(a) Treatment effect

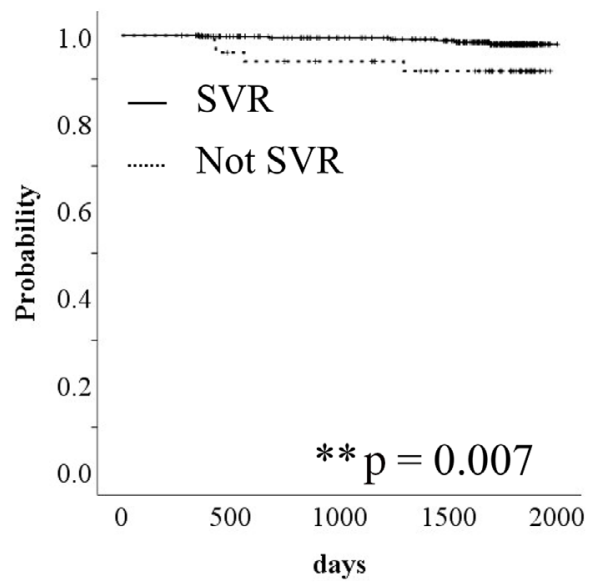

(b) History of HCC

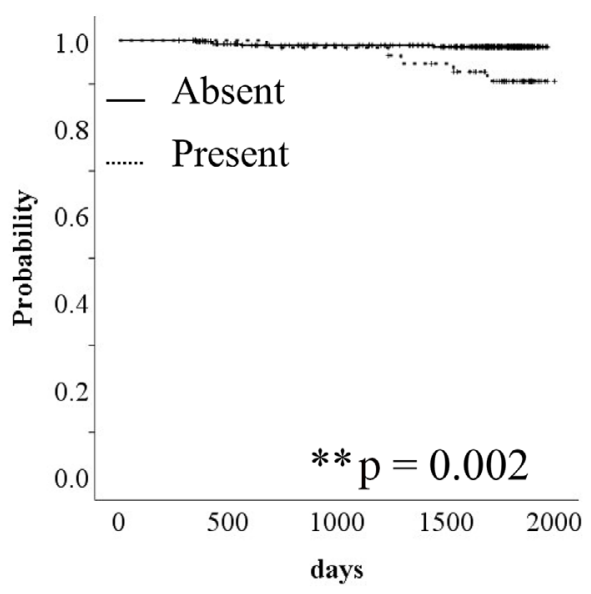

Figure 5. Liver disease-related mortality. (a) Significantly reduced number of liver disease-related deaths in the treatment effect of an SVR group ( $p=0.007)$. (b) The rate of liver disease-related deaths was significantly worse in the group with a history of HCC than in the group without such a history (p=0.002). SVR: sustained viral response, HCC: hepatocellular carcinoma

2007 and 2011 to serve as centers of excellence for hepatitis treatment in the region and to improve access to hepatitis care by organizing training sessions for specialists and nonspecialists (33). In our prefecture, regional core centers are cooperating with each other to provide medical care for liver diseases, and DAA treatment is also actively being performed in non-specialized medical institutions. There is no significant difference in the actual treatment outcome between general medical institutions and specialized medical institutions, suggesting that medical care for hepatitis $\mathrm{C}$ is being performed correctly in a wide range of fields.

Several limitations associated with the present study warrant mention. First, it is a retrospective study in which the methods of follow-up and liver cancer screening were not consistent, and differences are expected among institutions. Second, we were unable to track the extent to which pa- tients had been referred to other hospitals after treatment. Finally, the study was associated with some limitations, and the evaluation of the cancer risk after DAA treatment was therefore incomplete.

In conclusion, there is a risk of hepatocarcinogenesis even after achieving an SVR with DAA, so follow-up is necessary. In this study, the majority of patients seem to have received appropriate follow-up after DAA treatment, including monitoring for hepatocarcinogenesis. However, in younger patients, the interruption of hospital visits was rather frequent, and this problem will likely need to be addressed in the future, including employment support.

The authors state that they have no Conflict of Interest (COI). 


\section{References}

1. Kiyosawa K, Sodeyama T, Tanaka E, et al. Interrelationship of blood transfusion, non-A, non-B hepatitis and hepatocellular carcinoma: analysis by detection of antibody to hepatitis $\mathrm{C}$ virus. Hepatology 12: 671-675, 1990.

2. Ogawa E, Furusyo N, Nakamuta M, et al. Glecaprevir and pibrentasvir for Japanese patients with chronic hepatitis $\mathrm{C}$ genotype 1 or 2 infection: results from a multicenter, real-world cohort study. Hepatol Res 49: 617-626, 2019.

3. Osawa M. Real-world efficacy of glecaprevir plus pibrentasvir for chronic hepatitis $\mathrm{C}$ patient with previous direct-acting antiviral therapy failures. 3-8, 2018.

4. Kusakabe A, Kurosaki M, Itakura J, et al. Efficacy and safety of glecaprevir/pibrentasvir as retreatment therapy for patients with genotype 2 chronic hepatitis $\mathrm{C}$ who failed prior sofosbuvir plus ribavirin regimen. Hepatol Res 49: 1121-1126, 2019.

5. D'Ambrosio R, Pasulo L, Puoti M, et al. Real-world effectiveness and safety of glecaprevir/pibrentasvir in 723 patients with chronic hepatitis C. J Hepatol 70: 379-387, 2019.

6. Toyoda H, Atsukawa M, Watanabe T, et al. Real-world experience of 12-week direct-acting antiviral regimen of glecaprevir and pibrentasvir in patients with chronic hepatitis $\mathrm{C}$ virus infection. $\mathrm{J}$ Gastroenterol Hepatol 1-7, 2019.

7. Persico M, Aglitti A, Milella M, et al. Real-life glecaprevir/pibrentasvir in a large cohort of patients with hepatitis $\mathrm{C}$ virus infection: the MISTRAL study. Liver Int 39: 1852-1859, 2019.

8. Ikeda K, Saitoh S, Arase Y, et al. Effect of interferon therapy on hepatocellular carcinogenesis in patients with chronic hepatitis type C: a long-term observation study of 1,643 patients using statistical bias correction with proportional hazard analysis. Hepatology 29: 1124-1130, 1999.

9. Cardoso AC, Moucari R, Figueiredo-Mendes C, et al. Impact of peginterferon and ribavirin therapy on hepatocellular carcinoma: incidence and survival in hepatitis $\mathrm{C}$ patients with advanced fibrosis. J Hepatol 52: 652-657, 2010.

10. Watanabe S, Enomoto $N$, Koike $K$, et al. Cancer preventive effect of pegylated interferon $\alpha-2 b$ plus ribavirin in a real-life clinical setting in Japan: PERFECT interim analysis. Hepatol Res 41: 955964, 2011.

11. Hiramatsu N, Oze T, Takehara T. Suppression of hepatocellular carcinoma development in hepatitis $\mathrm{C}$ patients given interferonbased antiviral therapy. Hepatol Res 45: 152-161, 2015.

12. Nagaoki Y, Aikata H, Nakano N, et al. Development of hepatocellular carcinoma in patients with hepatitis $\mathrm{C}$ virus infection who achieved sustained virological response following interferon therapy: a large-scale, long-term cohort study. J Gastroenterol Hepatol 31: 1009-1015, 2016.

13. Tada $\mathrm{T}$, Toyoda H, Yasuda $\mathrm{S}$, et al. Long-term prognosis of liver disease in patients with eradicated chronic hepatitis $\mathrm{C}$ virus: an analysis using a Markov chain model. Hepatol Res 50: 936-946, 2020.

14. Reig M, Mariño Z, Perelló $C$, et al. Unexpected high rate of early tumor recurrence in patients with $\mathrm{HCV}$-related HCC undergoing interferon-free therapy. J Hepatol 65: 719-726, 2016.

15. Conti F, Buonfiglioli F, Scuteri A, et al. Early occurrence and recurrence of hepatocellular carcinoma in HCV-related cirrhosis treated with direct-acting antivirals. J Hepatol 65: 727-733, 2016.

16. Ide $\mathrm{T}$, Koga $\mathrm{H}$, Nakano $\mathrm{M}$, et al. Direct-acting antiviral agents do not increase the incidence of hepatocellular carcinoma development: a prospective, multicenter study. Hepatol Int 13: 293-301, 2019.

17. Dang H, Yeo YH, Yasuda S, et al. Cure with interferon free DAA is associated with increased survival in patients with HCV related HCC from both East and West. Hepatology 2, 2019.

18. Janjua NZ, Wong S, Darvishian M, et al. The impact of SVR from direct acting antiviral and interferon-based treatments for $\mathrm{HCV}$ on hepatocellular carcinoma risk. J Viral Hepat 2020.

19. Ioannou GN, Green PK, Berry K. HCV eradication induced by direct-acting antiviral agents reduces the risk of hepatocellular carcinoma. J Hepatol 68: 25-32, 2018.

20. McDonald SA, Pollock KG, Barclay ST, et al. Real-world impact following initiation of interferon-free hepatitis $\mathrm{C}$ regimens on liver-related outcomes and all-cause mortality among patients with compensated cirrhosis. J Viral Hepat 27: 270-280, 2020.

21. Kumada H, Suzuki Y, Ikeda K, et al. Daclatasvir plus asunaprevir for chronic HCV genotype $1 \mathrm{~b}$ infection. Hepatology 59: 20832091, 2014.

22. Suzuki Y, Ikeda K, Suzuki F, et al. Dual oral therapy with daclatasvir and asunaprevir for patients with HCV genotype $1 \mathrm{~b}$ infection and limited treatment options. J Hepatol 58: 655-662, 2013.

23. Tojima H, Kakizaki S, Takakusagi S, et al. Favorable outcome of retreatment by direct-acting antivirals for hepatitis $\mathrm{C}$ patients with daclatasvir plus asunaprevir combination therapy failure. Hepatol Res 50: 303-312, 2020.

24. Sterling RK, Lissen E, Clumeck N, et al. Development of a simple noninvasive index to predict significant fibrosis in patients with HIV/HCV coinfection. Hepatology 43: 1317-1325, 2006.

25. Sangiovanni A, Manini MA, Iavarone $M$, et al. The diagnostic and economic impact of contrast imaging techniques in the diagnosis of small hepatocellular carcinoma in cirrhosis. Gut 59: 638-644, 2010 .

26. Kanda Y. Investigation of the freely available easy-to-use software "EZR" for medical statistics. Bone Marrow Transplant 48: 452458, 2013.

27. Oza N, Isoda H, Ono T, Kanto T. Current activities and future directions of comprehensive hepatitis control measures in Japan: the supportive role of the Hepatitis Information Center in building a solid foundation. Hepatol Res 47: 487-496, 2017.

28. Roche B, Coilly A, Duclos-Vallee JC, Samuel D. The impact of treatment of hepatitis $\mathrm{C}$ with DAAs on the occurrence of HCC. Liver Int 38: 139-145, 2018.

29. Ioannou GN, Beste LA, Green PK, et al. Increased risk for hepatocellular carcinoma persists up to 10 years after $\mathrm{HCV}$ eradication in patients with baseline cirrhosis or high FIB-4 scores. Gastroenterology 157: 1264-1278.e4, 2019.

30. Darvishian M, Wong S, Binka M, et al. Loss to follow-up: a significant barrier in the treatment cascade with direct-acting therapies. J Viral Hepat 27: 243-260, 2020.

31. Wada K. The history and current state of drug abuse in Japan. Ann N Y Acad Sci 1216: 62-72, 2011.

32. Chung H, Ueda T, Kudo M. Changing trends in hepatitis $C$ infection over the past 50 years in Japan. Intervirology 53: 39-43, 2010 .

33. Setoyama H, Korenaga M, Kitayama Y, Oza N, Masaki N, Kanto T. Nationwide survey on activities of regional core centers for the management of liver disease in Japan: cumulative analyses by the Hepatitis Information Center 2009-2017. Hepatol Res 50: 165-173, 2020 .

The Internal Medicine is an Open Access journal distributed under the Creative Commons Attribution-NonCommercial-NoDerivatives 4.0 International License. To view the details of this license, please visit (https://creativecommons.org/licenses/ by-nc-nd/4.0/).

(C) 2021 The Japanese Society of Internal Medicine Intern Med 60: 3061-3070, 2021 